\title{
Modulation of sulfur metabolism enables efficient glucosinolate engineering
}

Morten E Møldrup ${ }^{1,2+}$, Fernando Geu-Flores ${ }^{1,2 \dagger}$, Carl E Olsen ${ }^{2,3}$, Barbara A Halkier ${ }^{1,2^{*}}$

\begin{abstract}
Background: Metabolic engineering in heterologous organisms is an attractive approach to achieve efficient production of valuable natural products. Glucosinolates represent a good example of such compounds as they are thought to be the cancer-preventive agents in cruciferous plants. We have recently demonstrated that it is feasible to engineer benzylglucosinolate (BGLS) in the non-cruciferous plant Nicotiana benthamiana by transient expression of five genes from Arabidopsis thaliana. In the same study, we showed that co-expression of a sixth Arabidopsis gene, $\gamma$-glutamyl peptidase 1 (GGP1), resolved a metabolic bottleneck, thereby increasing BGLS accumulation. However, the accumulation did not reach the expected levels, leaving room for further optimization.

Results: To optimize heterologous glucosinolate production, we have in this study performed a comparative metabolite analysis of BGLS-producing $N$. benthamiana leaves in the presence or absence of GGP1. The analysis revealed that the increased BGLS levels in the presence of GGP1 were accompanied by a high accumulation of the last intermediate, desulfoBGLS, and a derivative thereof. This evidenced a bottleneck in the last step of the pathway, the transfer of sulfate from 3'-phosphoadenosine-5'-phosphosulfate (PAPS) to desulfoBGLS by the sulfotransferase AtSOT16. While substitution of AtSOT16 with alternative sulfotransferases did not alleviate the bottleneck, experiments with the three genes involved in the formation and recycling of PAPS showed that co-expression of adenosine 5'-phosphosulfate kinase 2 (APK2) alone reduced the accumulation of desulfoBGLS and its derivative by more than $98 \%$ and increased BGLS accumulation 16-fold.

Conclusion: Adjusting sulfur metabolism by directing sulfur from primary to secondary metabolism leads to a remarkable improvement in BGLS accumulation and thereby represents an important step towards a clean and efficient production of glucosinolates in heterologous hosts. Our study emphasizes the importance of considering co-substrates and their biological nature in metabolic engineering projects.
\end{abstract}

\section{Background}

The plant kingdom is an extensive source of valuable compounds with a wide range of applications, most notably, in medicine. However, the availability of phytochemicals in their natural sources is often limited. In recent years, the efficient production of bioactive plant natural products has mainly been attempted through the metabolic engineering of microorganisms, as exemplified by the successful production of artemisinic acid in yeast [1]. Although microorganisms are generally considered easier to engineer, plants themselves present a series of

\footnotetext{
* Correspondence: bah@life.ku.dk

+ Contributed equally

'Department of Plant Biology and Biotechnology, Faculty of Life Sciences, University of Copenhagen, Thorvaldsensvej 40, DK-1871 Frederiksberg C, Denmark

Full list of author information is available at the end of the article
}

advantages as production organisms. For example, they only require soil, water, and sun light for their growth (which is unarguably $\mathrm{CO}_{2}$ friendly), and their care does not need highly educated personnel or specialized equipment. Therefore, there is a growing interest in the metabolic engineering of plants for the production of high-value bioactive compounds. Still, pathway engineering in plants is in its infancy and improvements are needed to reach the same level of flux control and yield optimization as seen in microorganisms. Part of the explanation for this is that stable plant transformations are notoriously time-consuming, making the challenge of stably engineering a whole biosynthetic pathway without prior confirmation of feasibility - an endeavour beyond the scope of most research projects.

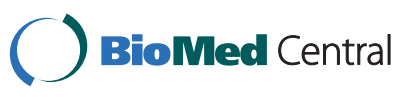


To obtain fast answers about the feasibility of engineering projects, we have turned to an established protocol for transient transformation of Nicotiana benthamiana [2], which has allowed us to rapidly probe the engineering of the sulfur-rich phytochemicals called glucosinolates (GLS). Apart from being well known as biopesticides, glucosinolates are thought to be the cancer-preventive agents in cruciferous plants [3]. Recently, we demonstrated the production of benzylglucosinolate (BGLS) in leaves of $N$. benthamiana by transient co-expression of five known biosynthetic genes from Arabidopsis thaliana [4]. The accumulation of BGLS was, however, concomitant with a disproportionately higher accumulation of a putative intermediate, the glutathione conjugate GS-B ( 100-fold higher at $6 \mathrm{dpi})$. This problem was solved by the discovery of $\gamma$-glutamylpeptidase 1 (GGP1), which was able to cleave GS-B in vitro, and whose presence in BGLS-producing $N$. benthamiana leaves led to a strong reduction in GS-B accumulation (> 99\% at $6 \mathrm{dpi}$ ) and a substantial increase in BGLS levels ( 4-fold at $6 \mathrm{dpi}$ ) [4]. The discovery of GGP1 showed that the transient system was not only useful for assessing the feasibility of engineering a given pathway, but also for gene discovery.

Although the presence of GGP1 in BGLS-producing $N$. benthamiana leaves resolved a major metabolic bottleneck, the increase in accumulation of BGLS did not account for the reduction in GS-B accumulation. In fact, only a small portion of the cleaved GS-B was converted to BGLS [4]. This suggested that an additional downstream bottleneck was present, and that BGLS production in this system could be further improved. In the present study, we report the identification of this bottleneck and its alleviation, which led to greatly increased BGLS levels. Our results are discussed in terms of the heterologous production of glucosinolates and in the general context of pathway engineering.

\section{Methods}

\section{Generation of plant expression constructs}

All constructs were assembled in Escherichia coli strain DH10B by USER cloning of coding sequences (CDSs) into the plasmid pCAMBIA3300-35Su [5]. The CDS of SAL1 (At5g63980) was amplified by PCR from cDNA made from leaves of $A$. thaliana Col-0. The CDSs of the remaining genes were amplified from existing cDNA clones: APK2 (At4g39940) from ABRC clone u21470; ATPS1 (At3g22890) from ABRC clone u10843; UGT74B1SUR1 (At1g24100-At2g20610) from ORF2nat [6]; and finally, AtSOT16 (At1g74100), AtSOT17 (At1g18590), AtSOT18 (Ag1g74090), and PAPS-S from published cDNA clones $[7,8]$. AtSOT16 has been previously referred to by us as AtST5a [4,6].

\section{Transient co-expression in leaves of $\mathbf{N}$. benthamiana}

Transient transformation of leaves of $N$. benthamiana was performed using Agrobacterium tumefaciens strain GV3850 and the silencing suppressor p19 [2]. Co-transformations were done by mixing different Agrobacterium strains (each carrying a single expression construct) in equal volumes prior to infiltration (Total $\mathrm{OD}_{600}$ of the mixed strains was 0.5), as previously described [4]. When comparing the effects of unequal number of strains, a strain transformed with an expression plasmid encoding for GFP [2] was included so as to obtain equal individual ODs across different strain combinations.

\section{GLS and LC-MS analysis}

Leaves were harvested at $6 \mathrm{dpi}$ by cutting four leaf discs from the Agrobacterium-infiltrated area and homogenizing them in $400 \mu \mathrm{l}$ of $\mathrm{MeOH}$ containing $0.02 \mathrm{mM}$ sinigrin as internal standard. Glucosinolates were quantified by HPLC-UV using the desulfoglucosinolate method [9] and BGLS amounts were calculated relative to sinigrin using a relative response factor of 0.8 [10]. Metabolite analysis was performed by LC-MS as previously described [4], and exact masses were obtained using a micrOTOF-Q detector (Bruker Daltonics).

\section{Endogenous N. benthamiana sulfatase activity assay}

$N$. benthamiana leaves infiltrated with Agrobacterium harboring AtSOT16 were harvested at $6 \mathrm{dpi}$ and homogenized in protein extraction buffer [ $250 \mathrm{mM}$ sucrose, $100 \mathrm{mM}$ Tris- $\mathrm{HCl} \mathrm{pH}$ 7,5, $50 \mathrm{mM} \mathrm{NaCl}, 2 \mathrm{mM}$ EDTA, 5\% PVPP, $5 \mathrm{mM}$ DTT and $1 \mathrm{X}$ 'Complete Protease Inhibitor' (Roche Molecular Biochemicals)]. After centrifugation at $20000 \mathrm{~g}$ at $4^{\circ} \mathrm{C}$ for $20 \mathrm{~min}, 50 \mu \mathrm{g}$ of soluble protein (from the supernatant) was added to $200 \mu \mathrm{l}$ reaction buffer I [100 mM Tris- $\mathrm{HCl} \mathrm{pH}$ 8.0, $10 \mathrm{mM} \mathrm{MgCl}_{2}, 1 \mathrm{mM}$ PAP (3'-phosphoadenosine 5'-phosphate, Sigma-Aldrich) and $1 \mathrm{mM}$ BGLS (Calbiochem)]. The reaction mixtures were incubated at $25^{\circ} \mathrm{C}$ for one hour and stopped and extracted by addition of $400 \mu \mathrm{l}$ ethyl acetate. A fraction of the ethyl acetate phase was evaporated and resuspended in acetonitrile. Sulfatase activity, represented by the formation of desulfoBGLS, was quantified by HPLC-UV [9].

As a positive control for the extraction of active soluble proteins, a sulfotransferase assay was carried out in parallel by adding $50 \mu \mathrm{g}$ protein from $20000 \mathrm{~g}$ supernatant to $100 \mu \mathrm{l}$ of reaction buffer II $[100 \mathrm{mM}$ Tris- $\mathrm{HCl}$ pH 8.0, $10 \mathrm{mM} \mathrm{MgCl}_{2}, 0.1 \mathrm{mM}$ PAPS (3'-phosphoadenosine 5'-phosphosulfate, Calbiochem) and $0.1 \mathrm{mM}$ dBGLS (obtained by desulfation of BGLS)]. The assay mixtures were incubated at $25^{\circ} \mathrm{C}$ for one hour and stopped by addition of $400 \mu \mathrm{l}$ of methanol. Sulfotransferase activity, represented by the formation of BGLS, 
was quantified by HPLC-UV using the desulfoglucosinolate method [9].

\section{Results}

Metabolite analysis of BGLS-producing $N$. benthamiana

We performed LC-MS analyses of extracts from Agrobacterium-infiltrated $N$. benthamiana leaves producing BGLS. The analyses confirmed that, when GGP1 is present together with the rest of the BGLS enzymes (encoded by the 2A-polycistronic constructs ORF1 and ORF2) [4], the peak of the glutathione conjugate GS-B was almost completely eliminated (Figure 1A). However, two major peaks appeared instead (Figure 1A). Based on MS2 fragmentation patterns (Figure 1B) and exact mass determination (4-digit mass errors of $<1.6 \mathrm{ppm}$ ), these peaks were annotated as desulfoBGLS (dBGLS) and malonylated dBGLS (mdBGLS). The identity of the dBGLS peak was confirmed by comparison to an authentic standard (Figure 1B). Mean dBGLS content at 6 dpi was quantified to be $1.58 \pm 0.16 \mathrm{nmol} / \mathrm{mg}$ fresh weight ( $\mathrm{nmol} / \mathrm{mg} \mathrm{fw}$ ), which is $\sim$ five-fold higher than the corresponding BGLS concentration. The size of the mdBGLS peak was comparable to that of dBGLS (Figure 1A), but the lack of a standard prevented its quantification.

Malonylation of metabolites and xenobiotics is a general plant storage and detoxification reaction, and unspecific malonyltransferases have been previously characterized in Nicotiana tabacum [11]. The co-occurrence of dBGLS and mdBGLS could thus readily be explained as a consequence of malonylation of the accumulating dBGLS by an unspecific endogenous malonyltransferase. dBGLS itself is the last intermediate of the BGLS pathway (Figure 1A), hence the accumulation of both dBGLS and mdBGLS suggested that the last reaction of the pathway, i.e. incorporation of sulfate by the sulfotransferase AtSOT16, constituted a metabolic bottleneck. Alternatively, the presence of dBGLS in BGLS-producing $N$. benthamiana could be due to the desulfation of intact BGLS by an unspecific endogenous sulfatase. However, when we tested soluble protein extracts from $N$. benthamiana leaves for in vitro sulfatase activity against BGLS, we were not able to detect any dBGLS (data not shown). Therefore, we focused on the sulfotransferase reaction in the remaining experiments.

\section{Testing of alternative Arabidopsis desulfoglucosinolate sulfotransferases}

A. thaliana ecotype Col-0 has three desulfoglucosinolate sulfotransferases: AtSOT16, AtSOT17 and AtSOT18, all of which were reported to convert dBGLS into BGLS in vitro $[7,12]$. The sulfotransferase that we had previously chosen to be part of the $2 \mathrm{~A}$ multicistronic construct ORF2 was AtSOT16, which has the highest $k_{\text {cat }} / K_{\mathrm{m}}$ towards dBGLS [12]. However, since the preferred in vitro substrates do not always correspond to the preferred in vivo substrates, we tested the ability of AtSOT17 and AtSOT18 to support BGLS engineering. Moreover, the AtSOT16 derived from ORF2 carried an $\mathrm{N}$-terminal His-tag as well as 17 extra amino acids (from the 2A sequence) in its C-terminus [6]. As these additional amino acids might interfere with catalytic functions, we also tested a native version of AtSOT16 in parallel with native AtSOT17 and AtSOT18.

In order to have an AtSOT16-free version of ORF2, we generated the expression construct GT/SUR, which encoded only the glucosyltransferase UGT74B1 and the $C$-S lyase SUR1 (Figure 2A). In addition, three expression constructs encoding for, respectively, native AtSOT16, AtSOT17 and AtSOT18 were generated (Figure 2A) and individually co-infiltrated with ORF1 (coding for CYP79A2 and CYP83B1), GGP1 and GT/ SUR. We subsequently compared BGLS and dBGLS accumulation in leaves infiltrated with the different strain combinations. No difference was observed in BGLS accumulation, neither between the tagged (from ORF2) and the un-tagged AtSOT16, nor between the three AtSOT isoforms (Figure 2B). The level of dBGLS remained slightly less constant, but more than twice as high as the BGLS content in all combinations (Figure 2B), which demonstrated that the alternative sulfotransferases could not alleviate the metabolic bottleneck.

The negative control without any AtSOT gave only a $\sim 50 \%$ reduction in BGLS production when compared to all other combinations (Figure 2B). Though reduced, the production of $0.17 \pm 0.04 \mathrm{nmol} \mathrm{BGLS} / \mathrm{mg} \mathrm{fw}$ in the absence of an Arabidopsis sulfotransferase shows that endogenous $N$. benthamiana sulfotransferases were able to catalyze the reaction. This is in agreement with a previous result, where we showed that, upon in vivo feeding of phenylacetothiohydroximate (the penultimate intermediate in the BGLS pathway) to wildtype Nicotiana tabacum, BGLS was produced, thereby demonstrating that the two last steps in the pathway could be catalyzed in a Nicotiana species by endogenous glucosyltransferases and sulfotransferases [6]. This results supported the hypothesis that the late enzymes of glucosinolate biosynthesis were recruited from general detoxification pathways. Nevertheless, and similarly to the present sulfotransferase experiment in $N$. benthamiana, the presence of an AtSOT did improve the accumulation of BGLS significantly [6].

\section{PAPS as limiting co-substrate}

All known sulfotransferases utilize 3'-phosphoadenosine5'-phosphosulfate (PAPS) as the activated form of sulfate, and the last step of the glucosinolate pathway is no exception. Furthermore, sulfotransferase reactions not 
A

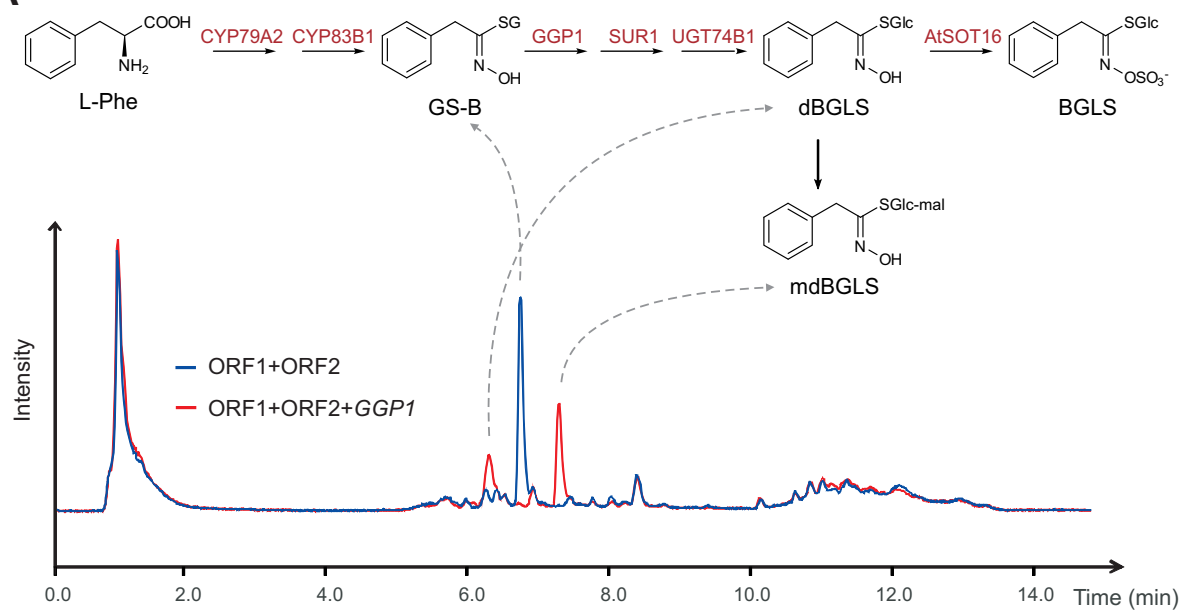

B
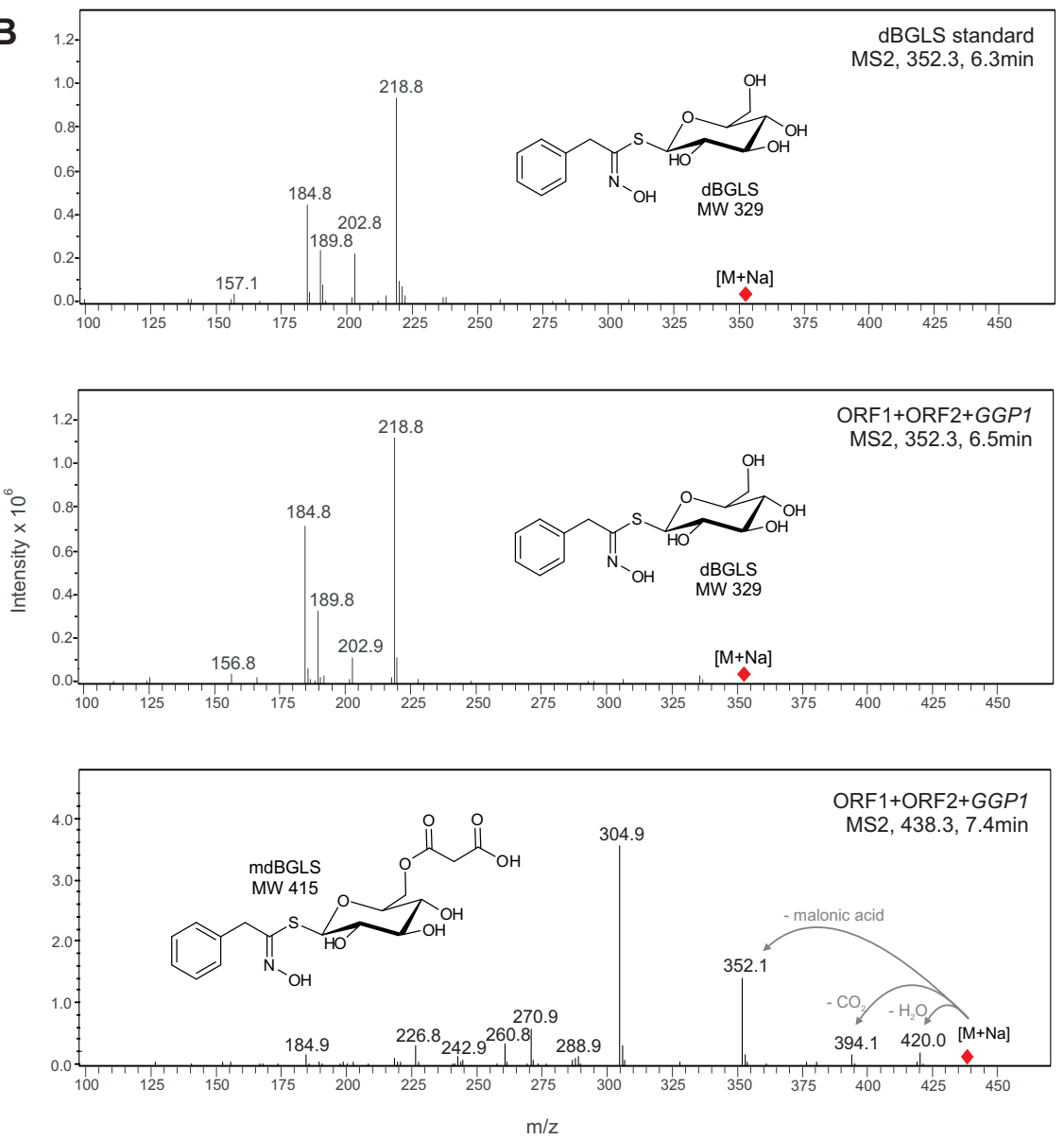

Figure 1 Metabolite analysis of BGLS-producing $\boldsymbol{N}$. benthamiana leaves. A) Total ion chromatograms from the LC-MS analysis of N. benthamiana leaves expressing ORF1 (coding for CYP79A2 and CYP83B1) and ORF2 (coding for SUR1, UGT74B1, and AtSOT16), in the presence or absence of GGP1 (red and blue traces, respectively). A scheme of the engineered pathway is inserted, where the identities of the three main chromatogram peaks have been assigned to by dashed arrows. Metabolite abbreviations are written in black, and enzyme names are written in red. B) MS2 fragmentation patterns of the $[\mathrm{M}+\mathrm{Na}]^{+}$adducts of standard dBGLS (upper panel) and of the prominent compounds eluting at 6.5 min (middle panel) and 7.4 min (lower panel) in the analysis of the extracts of $\mathrm{N}$. benthamiana leaves expressing ORF1, ORF2, and GGP1. The chemical structures of the proposed compounds are inserted. For mdBGLS, the location of the malonyl residue at position 6 is only tentative. 

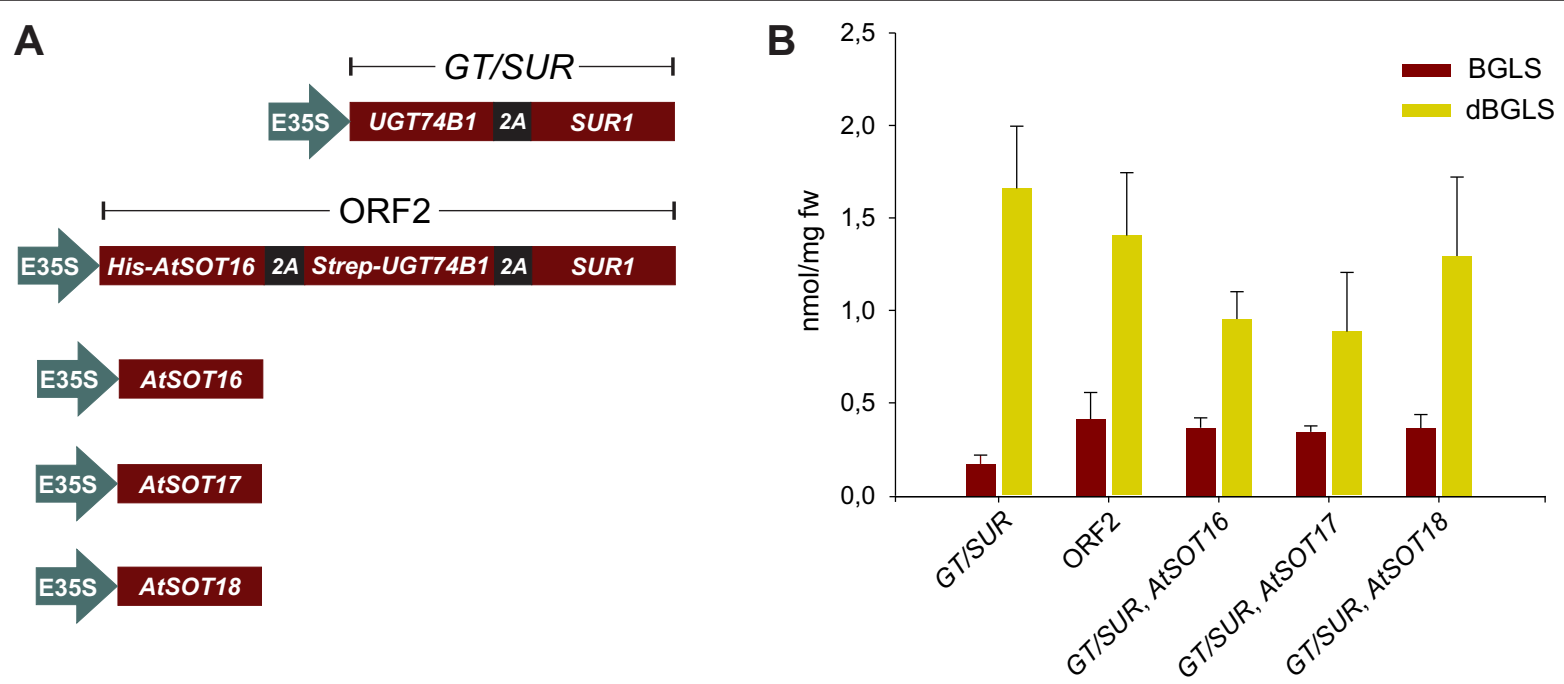

Figure 2 AtSOT-dependent BGLS and dBGLS accumulation. A) Expression constructs used for testing different versions of AtSOTs. His B) BGLS and dBGLS accumulation in leaves of $N$. benthamiana expressing ORF1 and GGP1 in combination with the indicated constructs at 6 dpi. Each data point represents the mean of eight biological replicates. Error bars represent standard deviations.

only produce the sulfated metabolite, but have also adenosine-3', 5'-bisphosphate (PAP) as a by-product.

PAPS is biosynthesized from adenosine-5'-triphosphate (ATP) and inorganic sulphate $\left(\mathrm{SO}_{4}{ }^{2-}\right)$ in two enzymatic steps. First, ATP sulfurylase (ATPS) sulfates ATP to form APS (adenosine 5'-phosphosulfate). Second, APS kinase (APK) phophorylates APS to form PAPS. The phosphate donor for this reaction is another molecule of ATP (Figure 3A) [13]. APK is located at an important metabolic branchpoint in sulfur assimilation, because a competing reaction, catalyzed by APS reductase, channels APS into the reductive assimilatory pathway leading to cysteine and, further downstream, to glutathione (Figure 3A). After sulfation, the by-product PAP is hydrolysed to AMP (adenosine-5'-monohosphate) by a bisphosphate nucleotidase. This reaction is biologically important not only because it removes PAP, which is an inhibitor of sulfotransferase reactions [12], but also because AMP can then be regenerated to form ATP, leading to actual recycling of the adenosine moiety of PAPS (Figure 3A). In Arabidopsis, the hydrolysis of PAP is most likely catalyzed by the multifunctional protein SAL1 [14].

In silico microarray-based co-expression databases such as ATTED-II [15] and CressExpress [16] are powerful tools for elucidating metabolic networks in Arabidopsis. We have previously used such databases to identify genes in the GLS pathway [4,9]. In ATTED-II, searches were performed using both SUR1 and CYP83B1 as query. ATP sulfurylase 1 (ATPS1), APS kinase 1 (APK1), APS kinase 2 (APK2) and SAL1, all of which are likely to be involved in the formation and recycling of PAPS, were among the top 24 co-expressed genes for both queries, in addition to many known GLS biosynthetic genes (data not shown). A similar picture was seen in CressExpress, where APK2, APK1 and $A T P S 1$ were among the top five co-regulated genes when five GLS biosynthetic genes (CYP79B2, CYP83B1, SUR1, UGT74B1, and AtSOT16) were used as query (data not shown). Therefore, we hypothesized that the lack of a highly efficient PAPS formation and recycling machinery in $N$. benthamiana prevented the sulfotransferase reaction from being carried to completion.

A relationship between APK genes and GLS biosynthesis was recently found by Mugford et al., who demonstrated that the Arabidopsis apk1/apk2 double knockout mutant had reduced levels of GLS and accumulated desulfoglucosinolates [17]. Furthermore, it has been shown that ATPS1, ATPS3, APK1, and APK2 are regulated by MYB transcription factors known to regulate both aliphatic and indolic glucosinolate biosynthesis [18]. This further suggested that the formation of PAPS could be limiting in BGLS-producing $N$. benthamiana leaves.

To test the hypothesis that an increased PAPS formation and recycling would aid BGLS production in leaves of $N$. benthamiana, we generated individual plant expression constructs carrying the native coding sequences of ATPS1, APK2 and SAL1, respectively. However, these three proteins carry predicted chloroplast targeting peptides (cTPs) and their fusions to fluorescent proteins have been shown to localize to chloroplasts $[17,19,20]$, whereas the glucosinolate pathway is proposed to be cytosolic [21]. This may pose a 


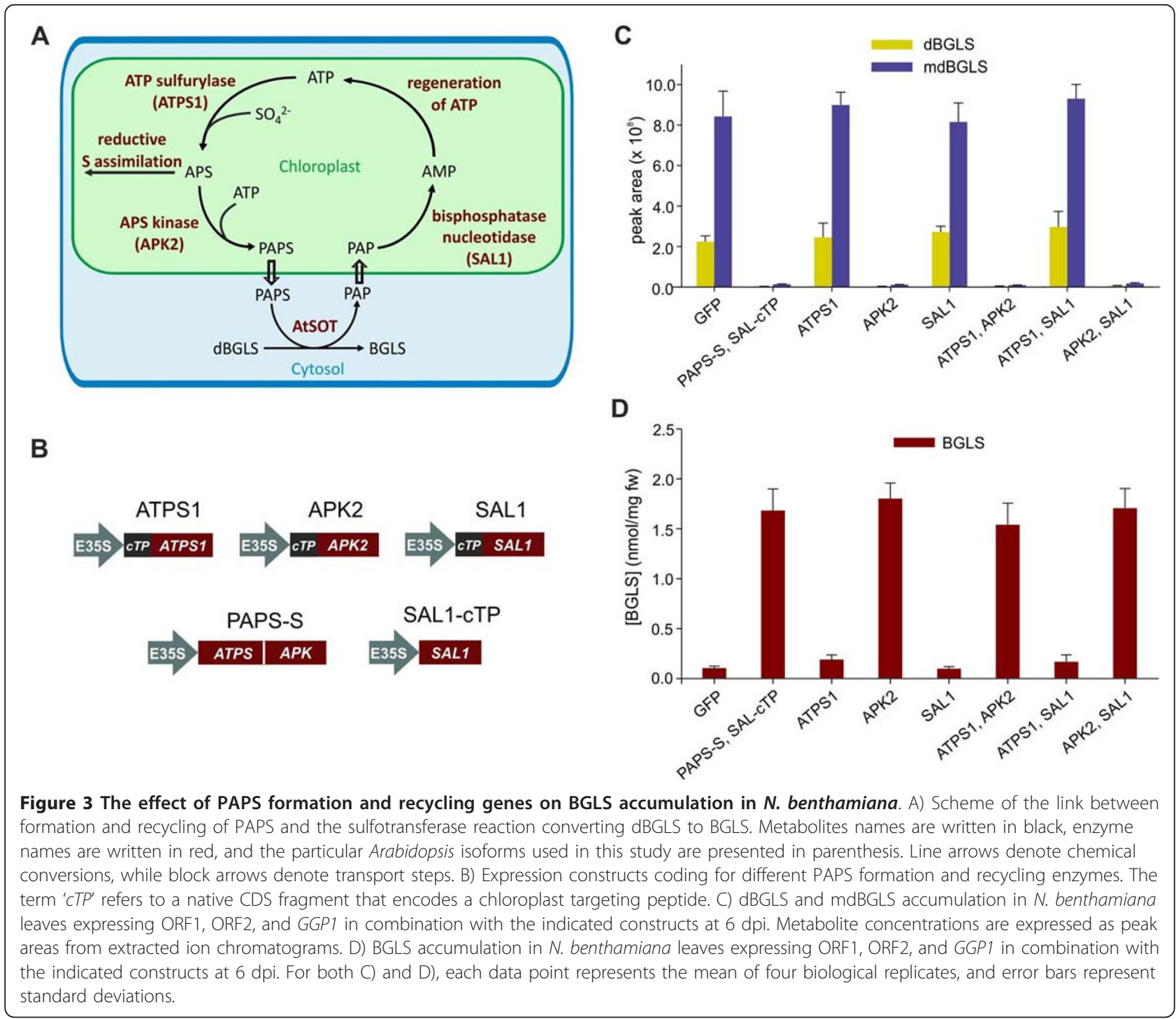

problem, as the co-substrate PAPS would have to be transported very efficiently from the chloroplast to the cytosol, while the by-product PAP would have to be mobilized efficiently in the opposite way. As a means to overcome this potential problem, we tested in parallel whether the PAPS formation and recycling machinery could be established in the cytosol. This was attempted using an ATPS-APK fusion protein from the marine worm Urechis caupo lacking a cTP, also referred to as PAPS synthetase (PAPS-S) [8], and a truncated version of SAL1 without its native cTP, which we named SAL1-cTP.

Agrobacterium strains harbouring the different PAPS formation and recycling genes (Figure $3 \mathrm{~B}$ ) were infiltrated into $N$. benthamiana leaves together with strains harbouring the BGLS biosynthetic genes, both individually and in selected combinations. In all experiments where APK2 was included, the levels of dBGLS and
mdBGLS were reduced > 98\% (Figure 3C). This was accompanied by a $\sim 16$-fold increase in mean BGLS levels (Figure 3D). ATPS1 and SAL1 did not affect the content of BGLS, dBGLS or mdBGLS any further, indicating that the endogenous ATPS and bisphosphate nucleotidase activities sufficiently support a highly increased PAPS formation and recycling, and that only the APK activity was limiting. The accumulation of BGLS, dBGLS, and mdBGLS in the presence of PAPS-S and SAL1-cTP, both lacking cTPs, did not differ from the accumulation seen with the plastid localized APK2. This suggests that the shuttling of PAPS and PAP across chloroplast membranes was not a limiting factor. In the presence of APK2, mean BGLS levels reached $1.80 \pm$ $0.16 \mathrm{nmol} / \mathrm{mg} \mathrm{fw}$ at $6 \mathrm{dpi}$. This is equivalent to the total GLS levels in rosette leaves of Arabidopsis Col-0 before bolting [22]. 


\section{Discussion}

In this study, we have successfully optimized the heterologous production of BGLS in leaves of $N$. benthamiana, reaching glucosinolate levels that are equal or higher than those observed in plants that produce glucosinolates naturally. The optimization was enabled by the identification of a bottleneck in the last biosynthetic reaction, the transfer of sulfate to dBGLS by a sulfotransferase. The bottleneck was resolved solely by co-production of APK2, an enzyme that converts the intermediate APS in the sulfur assimilatory pathway into the sulfotransferase co-substrate, PAPS.

In mammalian systems, where numerous xenobiotics follow sulfation-dependet detoxification routes, sulfate transfer has been described as a high-affinity, low-capacity process [23]. The identification of the sulfotransferase bottleneck in our engineered BGLS pathway indicates that sulfate transfer is also a lowcapacity process in non-cruciferous plants. However, the exact reasons for the low capacity are different. In rats and mice, the limiting factors appear to be the availability of sulfate and the sulfotransferases themselves [23]. Because the sulfotransferase problem in $N$. benthamiana was completely solved by co-expressing $A P K 2$, the provision of PAPS was clearly the limiting factor (and not sulfate availability nor the sulfotransferase AtSOT16).

Even under PAPS-limiting conditions, the addition of either AtSOT resulted in increased BGLS accumulation as compared when only endogenous $N$. benthamiana sulfotransferases were available. This increase can be speculated to be even more pronounced under PAPS unlimited conditions (i.e. in the presence of APK2). Conversely, it was not possible to determine any difference in the performance of the three AtSOTs under PAPS-limiting conditions, and future studies could therefore focus on determining the optimal AtSOT for production of specific GLS in the presence of $A P K 2$.

The finding that APK2 alone could resolve the sulfotransferase bottleneck also demonstrates that other PAPS-related activities, namely ATPS and bisphosphate nucleotidase activities were not limiting either. This likely reflects that the two latter enzymes participate in important PAPS-independent pathways. ATPS synthesizes the central sulfur assimilation intermediate APS, which can either be used in the reductive sulfur assimilation pathway leading to cysteine and glutathione or converted into to PAPS by an APK. In turn, bisphosphate nucleotidases not only hydrolyze PAP, but also inositol polyphosphate [14] and have been linked to numerous physiological processes such as salt, cold, and drought tolerance [24], RNA silencing [25], and leaf morphogenesis [26].

Although usually drawn as a linear pathway, the glucosinolate pathway is fueled by the co-substrates NADPH, glutathione, UDP-glucose, and PAPS [21]. While the three former co-substrates are used in many ubiquitous processes in primary metabolism, most sulfation processes are considered part of secondary metabolism [17]. Therefore, the formation and regeneration of $\mathrm{NADPH}$, glutathione, and UDP-glucose is expected to be under more stringent control than that of PAPS (for example, by strict feedback regulation). This helps rationalizing why efficient glucosinolate production in $N$. benthamiana does not seem to require the engineering of co-substrates other than PAPS.

\section{Conclusion}

Our study shows that modulation of sulfur metabolism towards enhanced PAPS biosynthesis enables an efficient heterologous production of glucosinolates in $N$. benthamiana. This represents an important step towards a clean and efficient production of glucosinolates in heterologous hosts and emphasizes the importance of considering co-substrates and their biological nature in metabolic engineering projects.

\section{Abbreviations}

BGLS: benzylglucosinolate; dBGLS: desulfobenzylglucosinolate; mdBGLS: malonyldesulfobenzylglucosinolate; PAPS,: 3'-phosphoadenosine 5'-phosphosulfate; PAP: 3'-phosphoadenosine 5'-phosphate; APS: adenosine 5'-phosphosulfate; ATPS ATP: sulfurylase; APK, APS: kinase; PAPS-S, PAPS: synthetase.

\section{Acknowledgements}

We thank Dr. Markus Piotrowski (Ruhr-Universität, Bochum) and Dr. Thomas Leustek (Rutgers University, New Jersey) for kindly providing sulfotransferase and PAPS-S clones, respectively. Novozymes is acknowledged for the Novo Scholarship to M. E. Møldrup. This work was supported by the Villum Kann Rasmussen Fond through its support to the VKR Research Centre Pro-Active Plants.

\section{Author details}

${ }^{1}$ Department of Plant Biology and Biotechnology, Faculty of Life Sciences, University of Copenhagen, Thorvaldsensvej 40, DK-1871 Frederiksberg C, Denmark. ${ }^{2}$ VKR Research Centre for Pro-Active Plants, Faculty of Life Sciences, University of Copenhagen, Thorvaldsensvej 40, DK-1871 Frederiksberg C, Denmark. ${ }^{3}$ Department of Natural Sciences, Faculty of Life Sciences, University of Copenhagen, Thorvaldsensvej 40, DK-1871 Frederiksberg C, Denmark.

\section{Authors' contributions}

MEM participated in the design of the study, carried out most of the experimental work and drafted the manuscript. FG participated in the design of the study, helped carrying out the experimental work, and contributed to manuscript drafting and revising. CEO performed the LC-MS. BAH was overall study director and supervisor, participated in study design, helped to revise the manuscript and obtained the funding. All authors read and approved the final version.

Received: 18 June 2010 Accepted: 31 January 2011 Published: 31 January 2011 


\section{References}

1. Ro DK, Paradise EM, Ouellet M, Fisher KJ, Newman KL, Ndungu JM, Ho KA Eachus RA, Ham TS, Kirby J, Chang MCY, Withers ST, Shiba Y, Sarpong R, Keasling JD: Production of the antimalarial drug precursor artemisinic acid in engineered yeast. Nature 2006, 440:940-943.

2. Voinnet $O$, Rivas $S$, Mestre $P$, Baulcombe $D$ : An enhanced transient expression system in plants based on suppression of gene silencing by the p19 protein of tomato bushy stunt virus. Plant J 2003, 33:949-956.

3. Traka M, Mithen R: Glucosinolates, isothiocyanates and human health Phytochemistry Reviews 2009, 8:269-282.

4. Geu-Flores F, Nielsen MT, Nafisi M, Møldrup ME, Olsen CE, Motawia MS, Halkier BA: Glucosinolate engineering identifies a [gamma]-glutamyl peptidase. Nat Chem Biol 2009, 5:575-577.

5. Nour-Eldin HH, Hansen BG, Norholm MHH, Jensen JK, Halkier BA: Advancing uracil-excision based cloning towards an ideal technique for cloning PCR fragments. Nucleic Acids Res 2006, 34

6. Geu-Flores F, Olsen CE, Halkier BA: Towards engineering glucosinolates into non-cruciferous plants. Planta 2009, 229:261-270.

7. Piotrowski M, Schemenewitz A, Lopukhina A, Muller A, Janowitz T, Weiler EW, Oecking C: Desulfoglucosinolate Sulfotransferases from Arabidopsis thaliana Catalyze the Final Step in the Biosynthesis of the Glucosinolate Core Structure. J Biol Chem 2004, 279:50717-50725.

8. Rosenthal $E$, Leustek T: A multifunctional Urechis caupo protein, PAPS synthetase, has both ATP sulfurylase and APS kinase activities. Gene 1995, 165:243-248.

9. Hansen BG, Kliebenstein DJ, Halkier BA: Identification of a flavinmonooxygenase as the S-oxygenating enzyme in aliphatic glucosinolate biosynthesis in Arabidopsis. Plant Journal 2007, 50:902-910.

10. Brown PD, Tokuhisa JG, Reichelt M, Gershenzon J: Variation of glucosinolate accumulation among different organs and developmental stages of Arabidopsis thaliana. Phytochemistry 2003, 62:471-481.

11. Taguchi G, Shitchi Y, Shirasawa S, Yamamoto H, Hayashida N: Molecular cloning, characterization, and downregulation of an acyltransferase that catalyzes the malonylation of flavonoid and naphthol glucosides in tobacco cells. Plant Journal 2005, 42:481-491.

12. Klein M, Reichelt M, Gershenzon J, Papenbrock J: The three desulfoglucosinolate sulfotransferase proteins in Arabidopsis have different substrate specificities and are differentially expressed. FEBS Journal 2006, 273:122-136.

13. Kopriva S: Regulation of sulfate assimilation in Arabidopsis and beyond. Ann Bot 2006, 97:479-495.

14. Quintero FJ, Garciadeblas B, Rodriguez NA: The SAL1 gene of Arabidopsis, encoding an enzyme with $3^{\prime}\left(2^{\prime}\right), 5^{\prime}$-bisphosphate nucleotidase and inositol polyphosphate 1-phosphatase activities, increases salt tolerance in yeast. Plant Cell 1996, 8:529-537.

15. Obayashi T, Kinoshita K, Nakai K, Shibaoka M, Hayashi S, Saeki M, Shibata D, Saito K, Ohta H: ATTED-II: a database of co-expressed genes and cis elements for identifying co-regulated gene groups in Arabidopsis. Nucleic Acids Res 2007, 35:D863-D869.

16. Srinivasasainagendra V, Page GP, Mehta T, Coulibaly I, Loraine AE: CressExpress: A tool for large-scale mining of expression data from Arabidopsis. Plant Physiol 2008, 147:1004-1016.

17. Mugford SG, Yoshimoto N, Reichelt M, Wirtz M, Hill L, Mugford ST, Nakazato Y, Noji M, Takahashi H, Kramell R, Gigolashvili T, Flugge UI, Wasternack C, Gershenzon J, Hell R, Saito K, Kopriva S: Disruption of Adenosine-5'-Phosphosulfate Kinase in Arabidopsis Reduces Levels of Sulfated Secondary Metabolites. Plant Cell 2009, 21:910-927.

18. Yatusevich R, Mugford SG, Matthewman C, Gigolashvili T, Frerigmann H, Delaney S, Koprivova A, Flugge UI, Kopriva S: Genes of primary sulfate assimilation are part of the glucosinolate biosynthetic network in Arabidopsis thaliana. Plant Journal 2010, 62:1-11.

19. Leustek T, Murillo M, Cervantes M: Cloning of A Cdna-Encoding Atp Sulfurylase from Arabidopsis-Thaliana by Functional Expression in Saccharomyces-Cerevisiae. Plant Physiol 1994, 105:897-902.

20. Rodriguez VM, Chetelat A, Majcherczyk P, Farmer EE: Chloroplastic Phosphoadenosine Phosphosulfate Metabolism Regulates Basal Levels of the Prohormone Jasmonic Acid in Arabidopsis Leaves. Plant Physiol 2010, 152:1335-1345.

21. Sønderby I, Geu-Flores F, Halkier BA: Biosynthesis of glucosinolates - gene discovery and beyond. Trends in Plant Science 2010, 15:283-290.
22. Petersen BL, Chen SX, Hansen $\mathrm{CH}$, Olsen $\mathrm{CE}$, Halkier BA: Composition and content of glucosinolates in developing Arabidopsis thaliana. Planta 2002, 214:562-571.

23. Klaassen CD, Boles JW: Sulfation and sulfotransferases .5. The importance of 3 '-phosphoadenosine $5^{\prime}$-phosphosulfate (PAPS) in the regulation of sulfation. Faseb Journal 1997, 11:404-418.

24. Xiong L, Lee Bh, Ishitani M, Lee H, Zhang C, Zhu JK: FIERY1 encoding an inositol polyphosphate 1-phosphatase is a negative regulator of abscisic acid and stress signaling in Arabidopsis. Genes \& Development 2001, 15:1971-1984.

25. Gy I, Gasciolli V, Lauressergues D, Morel JB, Gombert J, Proux F, Proux C, Vaucheret H, Mallory AC: Arabidopsis FIERY1, XRN2, and XRN3 Are Endogenous RNA Silencing Suppressors. Plant Cell 2007, 19:3451-3461.

26. Robles P, Fleury D, Candela H, Cnops G, Alonso-Peral MM, Anami S, Falcone A, Caldana C, Willmitzer L, Ponce MR, Van Lijsebettens M, Micol LL: The RON1/FRY1/SAL1 Gene Is Required for Leaf Morphogenesis and Venation Patterning in Arabidopsis. Plant Physiol 2010, 152:1357-1372.

doi:10.1186/1472-6750-11-12

Cite this article as: Møldrup et al:: Modulation of sulfur metabolism enables efficient glucosinolate engineering. BMC Biotechnology 2011 $11: 12$.

\section{Submit your next manuscript to BioMed Central and take full advantage of:}

- Convenient online submission

- Thorough peer review

- No space constraints or color figure charges

- Immediate publication on acceptance

- Inclusion in PubMed, CAS, Scopus and Google Scholar

- Research which is freely available for redistribution

Submit your manuscript at www.biomedcentral.com/submit
C) Biomed Central 